\title{
Neurobehavioral recovery in patients who emerged from prolonged disorder of consciousness: a retrospective study
}

\author{
Hoo Young Lee ${ }^{1,2,3}$, Jung Hyun Park ${ }^{3,4}$, Ae Ryoung Kim ${ }^{5}$, Misun Park ${ }^{6}$ and Tae-Woo Kim,2*
}

\begin{abstract}
Background: We investigated the clinical course of patients with prolonged disorders of consciousness (PDoC), predictors of emergence from PDoC (EDoC), and the temporal dynamics of six neurobehavior domains based on the JFK Coma Recovery Scale-Revised (CRS-R) during the recovery.

Methods: A total of 50 traumatic and non-traumatic patients with PDoC were enrolled between October 2014 and February 2017. A retrospective analysis of the clinical findings and neurobehavioral signs was conducted using standardized methodology such as CRS-R. The findings were used to investigate the incidence and predictors of EDoC and determine the cumulative pattern of neurobehavioral recovery at 6 months, 1 year, and 2 years post-injury.

Results: The results showed that 46\% of the subjects emerged from PDoC after 200 median days (64-1197 days) of injury onset. The significant predictors of EDoC included minimally conscious state (MCS) (vs. vegetative state), higher auditory, communication, arousal, total CRS-R scores, shorter lag time post-injury, and the absence of intra-axial lesions. In terms of cumulative recovery of motor and communication signs in patients who emerged from PDoC, 39 and 32\% showed EDoC at 6 months post-injury, and 88 and 93\% exhibited EDoC at 2 years post-injury, respectively.

Conclusions: Nearly half of the patients with PDoC recovered consciousness during inpatient rehabilitation. MCS, shorter lag time, the absence of intra-axial lesions, higher auditory, communication, arousal, and total CRS-R scores were important predictors for EDoC. Motor scores in the early stage of recovery and communication scores after prolonged intervals contributed to the higher levels of cumulative EDoC.
\end{abstract}

Keywords: Brain injuries, Consciousness disorders, Rehabilitation, Treatment outcome

\section{Background}

Disorders of consciousness (DoC), including vegetative state/unresponsive wakefulness syndrome (VS/UWS) and minimally conscious state (MCS), indicate a continuum of disruption in the arousal and awareness systems of the brain caused by severe acquired brain injury (ABI) [1-4].

\footnotetext{
*Correspondence: taewookimmd@gmail.com; drcadaver@naver.com

${ }^{1}$ TBI rehabilitation center, National Traffic Injury Rehabilitation Hospital, 260, Jungang-ro, Dogok-ri, Yangpyeong-eup, Yangpyeong-gun, Gyeonggi-do 12564, South Korea

${ }^{2}$ Department of Rehabilitation Medicine, Seoul National University Hospital, Seoul University College of Medicine, Seoul, South Korea

Full list of author information is available at the end of the article
}

VS/UWS is characterized by a lack of response to the environment, but spontaneous eye-opening along with evidence of sleep-wake cycles. In contrast, patients in MCS may demonstrate inconsistent but reproducible signs of awareness. Patients with prolonged $\mathrm{DoC}$ (PDoC) remain in VS/UWS or MCS for more than 4 weeks [5]. The US Aspen Workgroup proposed that emergence from DoC is characterized by reliable and consistent displays of functional communication with or without the functional use of objects [1-4].

The number of studies regarding the natural course of DoC after an ABI has grown over more than the last

(C) The Author(s). 2020 Open Access This article is licensed under a Creative Commons Attribution 4.0 International License, which permits use, sharing, adaptation, distribution and reproduction in any medium or format, as long as you give appropriate credit to the original author(s) and the source, provide a link to the Creative Commons licence, and indicate if changes were made. The images or other third party material in this article are included in the article's Creative Commons licence, unless indicated otherwise in a credit line to the material. If material is not included in the article's Creative Commons licence and your intended use is not permitted by statutory regulation or exceeds the permitted use, you will need to obtain permission directly from the copyright holder. To view a copy of this licence, visit http://creativecommons.org/licenses/by/4.0/. The Creative Commons Public Domain Dedication waiver (http://creativecommons.org/publicdomain/zero/1.0/) applies to the data made available in this article, unless otherwise stated in a credit line to the data. 
decade. Specifically, those with traumatic etiologies and diagnosis of MCS (as opposed to VS/UWS) at the time of rehabilitation admission have shown better prognoses, with regard to both the recovery of consciousness and the recovery of functional independence [6-9].

Even though studies have begun to demonstrate the recovery potential in certain subsets of patients with DoC, the outcomes and conclusions are comprehensibly heterogeneous across studies with rates of recovery of consciousness that range from 14 to 95\% [7]. Moreover, the nature, features, and prediction of the recovery process have not been fully elucidated.

These factors emphasize the need to investigate the clinical course and neurobehavioral recovery in patients who have emerged from DoC. Enhanced knowledge regarding the long-term outcome of individuals with $\mathrm{PDoC}$ may help clarify the range of outcomes expected after severe $\mathrm{ABI}$ and guide treatment decisions that reflect a more accurate assessment of patient prognosis.

It is very important to recognize changes and predict recovery from VS/UWS and MCS to emergence from $\mathrm{PDoC}$ in severely brain-injured patients who may be expected to survive their initial brain insults and transition through various states of impaired consciousness [10, 11]. It is especially important to understand the nature and course of neurobehavioral recovery based on the overall and hierarchical perspectives.

The aim of this study was to investigate the course and clinical characteristics of patients emerging from PDoC during neurorehabilitation and present a predictive model for the recovery of consciousness. In terms of tracking serial changes in the JFK Coma Recovery ScaleRevised (CRS-R), this study was the first of its kind to investigate the temporal dynamics of each neurobehavioral sign in CRS-R and their effects on the emergence from PDoC.

\section{Methods}

The study was a retrospective, observational study of patients with $\mathrm{PDoC}$ who were admitted to a comprehensive neurorehabilitation hospital in the Republic of Korea over a 3-year period from October 1, 2014, to February 28, 2017. The inpatient rehabilitation of patients with PDoC in Korea entails intense rehabilitative treatment for at least 3 hours each day during the first 2 years after onset, and about an hour and a half thereafter.

We retrospectively collected data from the Clinical Data Warehouse $(\mathrm{CDW})$ in the hospital, including a database of electronic medical records obtained from both inpatients and outpatients for real-time clinical analysis of the raw data with the approval of the Institutional Review Board of the National Traffic Injury Rehabilitation Hospital (No. NTRH-18005). The CDW contains almost all the medical records, including every field note of the medical staff (admission and discharge notes, progress reports, and nursing data), patient information data, and records (insurance, diagnostic codes, age, gender, and vital signs), test results (laboratory tests, functional assessments, and imaging studies) and treatment modalities (medications, therapies, and medical procedures). The IRB, in accordance with the Declaration of Helsinki, approved this study and granted waiver of consent because the data had been de-identified before they were used for the analysis of this study.

The inclusion criteria were patients of all ages with acquired traumatic or non-traumatic brain injury who were diagnosed with VS/UWS or MCS upon admission and based on serial evaluation data acquired during hospitalization. We confirmed the clinical diagnosis of VS/UWS, MCS, and EDoC based on CRS-R scores. Patients diagnosed with coma upon admission, exhibiting neurological or medical instability, and those without discharge evaluation were excluded. Patients with metabolic problems, which may provoke decreases in the level of consciousness, were also excluded (Fig. 1).

Even with various milestones of impaired consciousness, the patients were dichotomized into two groups depending on the emergence from $\mathrm{PDoC}$ during rehabilitation or persistent VS/UWS or MCS upon discharge.

During hospitalization, all patients underwent standardized and serial clinical evaluations for behavioral responsiveness. All patients were assessed with CRS-R upon admission and at a predetermined time, by a well-trained expert team composed of rehabilitation physicians and physical and occupational therapists who had more than 1 year of experience in evaluations. The evaluation of CRS$\mathrm{R}$ is based upon specific behavioral responses to sensory stimuli on 30 hierarchically arranged items administered in a standardized format. The lowest item on each subscale represents reflexive activity, whereas the highest items represent cognitive behaviors [12].

To determine the most consistent states of consciousness and rehabilitation outcomes, none of the centrally acting pharmacologic agents administered daily, such as antispasmodics, anticonvulsants, and neurostimulatory agents were withdrawn.

Along with pharmacological interventions, all patients received physical therapy and occupational therapy in a neurorehabilitation program for $3 \mathrm{~h}$ a day, 5 days a week. Whole-body vibration, neuromuscular electric stimulation, Bobath, kinesthetic stimulation, joint movement and range of motion exercise, mobility management, and tilt-table standing were provided by the physical therapists. Multisensory stimulation, sensory regulation or basal stimulation, familiar auditory sensory training and facio-oral stimulation techniques were provided by the occupational therapists. In all cases, the data were entered prospectively into the CDW because they were 


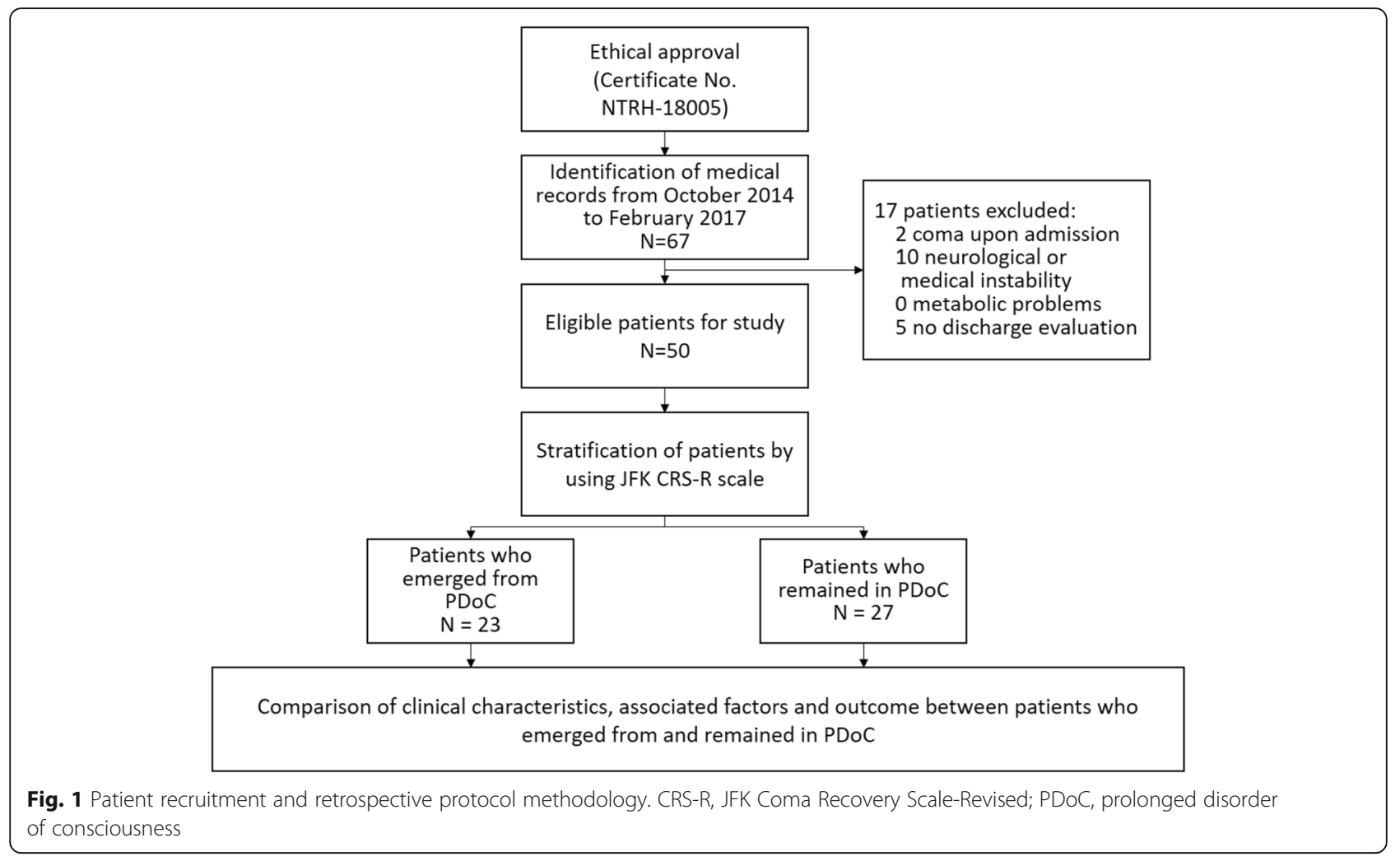

standardized test results. All the evaluators were blinded to the data used for advanced retrospective analysis.

After dividing the patients into two groups, the baseline characteristics, admission CRS-R scores, and 12 predictor variables associated with the incidence of consciousness recovery were investigated. The independent variables were as follows: 1) sex, 2) age at injury onset, 3) level of consciousness at admission (VS/UWS or MCS), 4) cause of the ABI (traumatic brain injury (TBI) or non-TBI), 5) the injury type (extra-axial or intra-axial lesion), 6) the lag time from the injury, 6) the CRS-R score at admission, 7) hydrocephalus, 8) ventriculoperitoneal shunt, 9) cranioplasty, 10) treatment with anticonvulsants (continued or discontinued), 11) seizure events, and 12) the level of education $(<12$ years or $\geq 12$ years). Further, we compared the degree of advancement in each sign of CRS-R during inpatient rehabilitation. Finally, we analyzed the temporal dynamics of auditory, visual, motor, oromotor, communication, and arousal scores and compared their effects on neurobehavioral recovery.

The baseline differences between the two subgroups were analyzed by the Wilcoxon rank-sum test for continuous and ordinal variables, and the chi-squared test or Fisher's exact test for categorical variables. The predictors of $\mathrm{EDoC}$ were analyzed by the univariate Cox proportional hazards model. The adjusted multivariate Cox proportional hazards model was used to investigate the optimal prediction parameters for EDoC. Maximally selected rank statistics were used to estimate the optimal cutoff value.

Kaplan-Meier plots were used to identify the median days at which each subscale of the CRS-R showed improvement of 1 point or more, and motor and communication scores reflecting EDoC. The plots were then converted to cumulative probabilities of attaining at least $10 \%$ progress in each sign and emergence in the motor and communication subscale at 6 months, 1 year, and 2 years post-injury.

Prognostic correlation between the CRS-R subscale scores and $\mathrm{EDoC}$ was analyzed using the marginal structural Cox model, after adjustment for time-varying confounders, such as clustered data on CRS-R at various time points from injury, during the longitudinal observation period.

Statistical analysis was performed using $\mathrm{R}$ software (version R.3.3.2; the R Foundation) and SAS version 9.4.

\section{Results}

\section{Patient clinical demographics}

Among the total of 1236 inpatients monitored during the 3year observation period, $40.9 \%(n=506)$ had acquired brain injuries as their main diagnosis. Of those patients, $13.2 \%$ $(n=67)$ were diagnosed with PDoC and 9.9\% $(n=50)$ referred for further analysis (Fig. 1) (Supplementary file 1).

The patients progressed through the stages of recovery at varying rates. Of the 50 patients, 25 were admitted 
with VS/UWS. Among the $12 \mathrm{VS} / \mathrm{UWS}$ patients who showed improvement in the level of consciousness, eight recovered to the MCS level and four demonstrated EDoC. Of the 25 patients who were admitted in MCSs, 19 emerged from an MCS. Overall, 46\% emerged from PDoC during inpatient rehabilitation. During the observational period, no patients died and no patient was lost to follow-up. The stimulant medications prescribed to the patients are summarized in Table 1.

The median (IQR) lag time from injury was 204.5 $(97.25,374.5)$ and the duration of inpatient rehabilitation was $92(62.5,121)$ days. In the recovery group, the emergence from PDoC occurred over a median period of 200 (129.5, 329, range 64-1197) days. Stratification of the recovery group based on diagnostic subtype (VS/UWS vs. MCS) indicated that patients with VS/UWS and MCS manifested EDoC in 164 (124, 236.25, range 112-345) and 209 (131.5, 346, range 64-1143) median days, respectively. Sub-group analysis of the participants by etiology (traumatic vs. non-traumatic) revealed that the patients with traumatic injury regained consciousness over a period of $158(124.25,292.5$, range $85-575)$ median days, and a median of 217 (154, 345, range 641143) days for non-traumatic injuries.

To investigate the prognostic outcomes in PDoC, the patients were retrospectively dichotomized into patients

Table 1 Summary of neuroplasticity stimulant drugs given to the patients with PDoC

\begin{tabular}{lcc}
\hline Prescribed Drugs & EDoC $(n=23)$ & PDoC $(n=27)$ \\
\hline $\begin{array}{l}\text { Noradrenergic } \\
\text { atomoxetine }\end{array}$ & 9 & 13 \\
$\begin{array}{l}\text { Dopaminergic } \\
\text { levodopa/carbidopa } \\
\text { methylphenidate }\end{array}$ & 18 & 17 \\
$\begin{array}{l}\text { Cholinergic } \\
\text { choline alfoscerate } \\
\text { donepezil } \\
\text { rivastigmine }\end{array}$ & 26 & \\
Serotonergic & & \\
escitalopram & & \\
paroxetine & & \\
sertraline & 9 & \\
Glutamatergic \\
memantine \\
Others \\
nicergoline \\
oxiracetam \\
zolpidem \\
$\begin{array}{l}\text { EDoC emergence from disorder of consciousness, PDoC prolonged disorder } \\
\text { of consciousness }\end{array}$
\end{tabular}

who emerged from PDoC and those who remained in PDoC states. Based on descriptive analysis, MCS (76\% vs. $24 \%, p<0.001)$, greater total CRS-R scores $(12.6 \pm$ 3.8 vs.6.1 $\pm 3.8, \mathrm{p}<0.001)$, extra-axial hemorrhage compared with intra-axial lesions $(87.5 \%$ vs. $12.5 \%, p=$ $0.014)$, and shorter lag time from injury $(219.1 \pm 232.3$ days vs. $321.5 \pm 266.2$ days, $p=0.048)$ were associated with emergence from PDoC (Table 2).

In terms of CRS-R scores, the admission scores on the auditory $(2.3 \pm 0.9$ vs. $1.1 \pm 1.0, \mathrm{p}<0.001)$, visual $(2.7 \pm$ 1.3 vs. $1.0 \pm 1.3, \mathrm{p}<0.001)$, motor $(3.4 \pm 1.4$ vs. $1.5 \pm 1.1$, $p<0.001)$, oromotor $(1.3 \pm 0.7$ vs. $0.7 \pm 0.7, p=0.002)$, communication $(0.7 \pm 0.5$ vs. $0.1 \pm 0.3, \mathrm{p}<0.001)$, and arousal $(2.1 \pm 0.7$ vs. $1.7 \pm 0.7, p=0.046)$ subscales were significantly higher in patients who emerged from PDoC compared with those who remained in PDoC states. Further, the degree of advancement in each CRS-R subscale during neurorehabilitation was significantly greater in the patients who emerged from PDoC compared with those who remained in PDoC states (Table 3) (Supplementary file 2).

\section{Optimal outcome prediction: variables and models}

Cox regression analysis was performed to investigate the significant predictors of emergence from PDoC. MCS and higher CRS-R scores at admission were significantly correlated with positive outcomes, whereas intra-axial brain lesions and prolonged lag time were significant predictors of negative outcomes (Table 1).

According to the multivariate Cox regression analysis and Akaike information criterion-based optimization, lag time and intra-axial lesions were significantly negatively correlated with emergence from PDoC.

In terms of the optimal cutoff value, lag days from the injury onset to neurorehabilitation within 528 days and total CRS-R scores greater than 6 were significantly associated with emergence from $\mathrm{PDoC}$.

Based on the neurobehavioral level upon admission, all the subscale scores in CRS-R significantly affected the emergence from PDoC. The arousal, communication, and auditory subscales were strongly correlated with emergence from MCS, followed by oromotor, visual, and motor subscales. The total CRS-R scores had the least impact (Table 4).

\section{Temporal dynamics of neurobehavioral signs during emergence from PDoC}

The median number of days required to advance at least one point in each CRS-R subscale was determined from Kaplan-Meier curves for the groups that emerged from PDoC. Among various recovery patterns, motor signs showed the most rapid recovery (191 median days), followed by auditory, arousal, communication, and visual scores in order. Oromotor scores showed the maximum improvement delay (284 median days) (Table 5). 
Table 2 Predictors of emergence from disorder of consciousness in univariate analysis

\begin{tabular}{|c|c|c|c|c|c|}
\hline Variable & $\mathrm{EDoC}(n=23)$ & $\operatorname{PDoC}(n=27)$ & $p$-value ${ }^{\dagger}$ & $\mathrm{HR}(95 \% \mathrm{Cl})^{\ddagger}$ & $p$-value \\
\hline \multicolumn{6}{|l|}{ Sex } \\
\hline Male & $16(50)$ & $16(50)$ & 0.645 & reference & \\
\hline Female & $7(38.9)$ & $11(61.1)$ & & $0.44(0.17,1.15)$ & 0.093 \\
\hline \multicolumn{6}{|l|}{ Age } \\
\hline Median (IQR) & $46(34,62.5)$ & $46(20,60)$ & 0.326 & $1.02(0.99,1.04)$ & 0.115 \\
\hline \multicolumn{6}{|l|}{ Level of consciousness } \\
\hline VS/UWS & $4(16)$ & $21(84)$ & $<0.001$ & reference & \\
\hline MCS & $19(76)$ & $6(24)$ & & $4.49(1.52,13.27)$ & 0.007 \\
\hline \multicolumn{6}{|l|}{ Total CRS-R score } \\
\hline Median (IQR) & $13(10,16)$ & $5(4,7.5)$ & $<0.001$ & $1.16(1.06,1.28)$ & 0.002 \\
\hline$\leq 6^{*}$ & $1(5.6)$ & $17(94.4)$ & & reference & \\
\hline$>6$ & $22(68.8)$ & $10(31.2)$ & & $10.02(1.34,74.61)$ & 0.028 \\
\hline \multicolumn{6}{|l|}{ Etiology } \\
\hline TBI & $14(48.3)$ & $15(51.7)$ & 0.927 & reference & \\
\hline Non-TBl & $9(42.9)$ & $12(57.1)$ & & $0.8(0.3,1.9)$ & 0.61 \\
\hline \multicolumn{6}{|l|}{ Injury Type $(n=46)$} \\
\hline Extra-axial hemorrhage & $7(87.5)$ & $1(12.5)$ & 0.014 & reference & \\
\hline Intra-axial lesion & $13(34.2)$ & $25(65.8)$ & & $0.09(0.03,0.24)$ & $<0.001$ \\
\hline \multicolumn{6}{|l|}{ Lag Time (days) } \\
\hline Median (IQR) & $133(86,212.5)$ & $222(126,443.5)$ & 0.048 & $0.99(0.98,0.99)$ & $<0.001$ \\
\hline$\leq 528^{*}$ & $21(48.8)$ & $22(51.2)$ & & reference & \\
\hline$>528$ & $2(28.6)$ & $5(71.4)$ & & $0.10(0.01,0.78)$ & 0.028 \\
\hline \multicolumn{6}{|l|}{ Hydrocephalus } \\
\hline Present & $11(40.7)$ & $16(59.3)$ & 0.6 & reference & \\
\hline Absent & $12(52.2)$ & $11(47.8)$ & & $1.77(0.78,4.06)$ & 0.174 \\
\hline \multicolumn{6}{|l|}{ VP shunt } \\
\hline Present & $8(50)$ & $8(50)$ & 0.932 & reference & \\
\hline Absent & $15(44.1)$ & $19(55.9)$ & & $1.28(0.54,3.04)$ & 0.573 \\
\hline \multicolumn{6}{|l|}{ Cranioplasty } \\
\hline Present & $10(43.5)$ & $13(56.5)$ & 0.964 & reference & \\
\hline Absent & $13(48.1)$ & $14(51.9)$ & & $1.69(0.73,3.91)$ & 0.218 \\
\hline \multicolumn{6}{|l|}{ Anticonvulsants } \\
\hline Continued & $15(45.5)$ & $18(54.5)$ & $>0.999$ & reference & \\
\hline Discontinued/not taking & $8(47.1)$ & $9(52.9)$ & & $1.19(0.5,2.86)$ & 0.694 \\
\hline \multicolumn{6}{|l|}{ Education $(n=19)$} \\
\hline$<12 \mathrm{yrs}$ & $3(25)$ & $9(75)$ & 0.156 & reference & \\
\hline$\geq 12 \mathrm{yrs}$ & $20(54.1)$ & $17(45.9)$ & & $1.95(0.58,6.6)$ & 0.282 \\
\hline
\end{tabular}

Values are presented as median (IQR) or number (\%)

CRS-R JFK Coma Recovery Scale-Revised, VS/UWS vegetative state/unresponsive wakefulness syndrome, MCS minimally conscious state, EDoC emergence from disorder of consciousness, PDoC prolonged disorder of consciousness, TBI traumatic brain injury, HR hazard ratio

*The optimal cutoff values of each variable were determined by maximally selected log-rank statistics

${ }^{\dagger} P$-value for the difference was determined by chi-squared, Fisher's exact, the Wilcoxon rank-sum tests

${ }^{\ddagger}$ Hazard ratio and $p$-value were calculated by univariate Cox proportional hazards regression

Meanwhile, the cumulative probabilities of at least one point of progress across the full range of each subscale showed a diverse pattern depending on the stage of recovery. At 180 days post-injury, the greatest cumulative probability of advancing one or more points was observed in the motor (43\%), visual (42\%), arousal (38\%), 
Table 3 Descriptive data for progress in CRS-R scores during neurorehabilitation

\begin{tabular}{|c|c|c|c|c|c|c|c|c|}
\hline \multirow{2}{*}{$\begin{array}{l}\text { Outcome } \\
\text { Measures }\end{array}$} & \multicolumn{2}{|c|}{ Emergence from PDoC } & \multirow{2}{*}{$\begin{array}{l}p^{-} \\
\text {value }\end{array}$} & \multicolumn{2}{|c|}{ Remain as PDoC } & \multirow{2}{*}{$\begin{array}{l}p^{-} \\
\text {value* }\end{array}$} & \multirow{2}{*}{$\begin{array}{l}p^{-} \\
\text {value }^{\dagger}\end{array}$} & \multirow{2}{*}{$\begin{array}{l}p^{-} \\
\text {value }^{\ddagger}\end{array}$} \\
\hline & Admission & $\overline{\text { Discharge }}$ & & Admission & $\overline{\text { Discharge }}$ & & & \\
\hline Auditory & $2(2,3)$ & $4(3.5,4)$ & $<0.001$ & $1(1,1)$ & $1(1,2)$ & 0.042 & $<0.001$ & $<0.001$ \\
\hline Visual & $3(2,4)$ & $4(4,5)$ & $<0.001$ & $1(0,1)$ & $1(1,3)$ & 0.011 & $<0.001$ & 0.004 \\
\hline Motor & $4(2,5)$ & $6(5,6)$ & $<0.001$ & $2(1,2)$ & $2(1,3)$ & 0.005 & $<0.001$ & 0.001 \\
\hline Oromotor & $1(1,2)$ & $2(2,3)$ & 0.001 & $1(0,1)$ & $1(1,1)$ & 0.001 & 0.002 & 0.041 \\
\hline Communication & $1(0,1)$ & $2(2,2)$ & $<0.001$ & $0(0,0)$ & $0(0,0)$ & 0.149 & $<0.001$ & $<0.001$ \\
\hline Arousal & $2(2,2.5)$ & $3(3,3)$ & $<0.001$ & $2(1,2)$ & $2(2,2)$ & 0.105 & 0.046 & 0.001 \\
\hline
\end{tabular}

Values are presented as median (IQR)

CRS-R JFK Coma Recovery Scale-Revised, PDoC prolonged disorder of consciousness

* Comparison between CRS-R scores at admission and discharge in each group

${ }^{+}$Comparison between admission CRS-R scores in the dichotomized groups

${ }^{\ddagger}$ Comparison between the degrees of advancement in the CRS-R scores in the dichotomized groups

auditory and communication (37\%, both), and oromotor (36\%) scores. At 2 years post-injury, the auditory score showed the highest cumulative probability of 95\%, followed by motor (93\%), visual and communication (92\% both), and arousal (90\%) scores, with the least probability in oromotor scores (76\%) (Table 5, Fig. 2).

We further investigated the temporal dynamics and cumulative probabilities of motor and communication scores associated with the following abilities: (1) functional use of objects, that is behavioral evidence of the ability to discriminate between at least two different objects and, (2) functional interactive communication, which may occur through verbalization, writing, 'yes' or 'no' signals, or the use of augmentative communication devices, which specifically correspond to EDoC (Fig. 3). Among 23 patients who manifested EDoC, 17 demonstrated $\mathrm{EDoC}$ via the functional use of objects at 209 (range 154-400) median days, whereas 18 showed EDoC via functional communication at 284 (range 150-390) median days. With regard to cumulative recovery, the functional use of objects was greater than the functional interaction at 180 days post-injury (32\% vs. 39\%). Eventually, the cumulative EDoC in the communication subscale increased and exceeded the motor subscale at 284 days post-injury. At 2 years post-injury, 93\% of the recovery group showed functional interaction while $88 \%$ demonstrated the functional use of objects.

\section{Discussion}

In this study, a retrospective observational analysis revealed a significant recovery of consciousness in patients with PDoC during inpatient rehabilitation, with $46 \%$ of the enrolled subjects emerging from PDoC. MCS, shorter lag time, the absence of intra-axial lesions, and higher auditory, communication, arousal, and total CRS$\mathrm{R}$ scores were important predictors of EDoC. The model

Table 4 CRS-R variables as predictors of emergence from disorder of consciousness

\begin{tabular}{|c|c|c|c|c|}
\hline Variable & $\mathrm{EDoC}(n=23)$ & PDoC $(n=27)$ & HR $(95 \%$ Cl) & $p$-value \\
\hline \multicolumn{5}{|l|}{ Auditory } \\
\hline Median (IQR) & $2(2,3)$ & $1(1,1)$ & $9.5(5.59,16.14)$ & $<0.001$ \\
\hline \multicolumn{5}{|l|}{ Visual } \\
\hline Median (IQR) & $3(2,4)$ & $1(0,1)$ & $3.78(2.92,4.90)$ & $<0.001$ \\
\hline \multicolumn{5}{|l|}{ Motor } \\
\hline Median (IQR) & $4(2,5)$ & $2(1,2)$ & $3.29(2.23,4.85)$ & $<0.001$ \\
\hline \multicolumn{5}{|l|}{ Oromotor } \\
\hline Median (IQR) & $1(1,2)$ & $1(0,1)$ & $4(2.35,6.81)$ & $<0.001$ \\
\hline \multicolumn{5}{|l|}{ Communication } \\
\hline Median (IQR) & $1(0,1)$ & $0(0,0)$ & $11.01(6.35,9.09)$ & $<0.001$ \\
\hline \multicolumn{5}{|l|}{ Arousal } \\
\hline Median (IQR) & $2(2,2.5)$ & $2(1,2)$ & $22.4(6.34,79.12)$ & $<0.001$ \\
\hline \multicolumn{5}{|l|}{ Total score } \\
\hline Median (IQR) & $13(10,16)$ & $5(4,8)$ & $1.59(1.41,1.8)$ & $<0.001$ \\
\hline
\end{tabular}

Values are presented as median (SD) or number (\%)

CRS-R JFK Coma Recovery Scale-Revised, EDoC emergence from prolonged disorder of consciousness, PDoC prolonged disorder of consciousness, HR hazard ratio 
Table 5 Median achievement time and cumulative probability to reach at least 1-point advancement

\begin{tabular}{|c|c|c|c|c|c|c|c|c|c|c|}
\hline \multirow{3}{*}{$\begin{array}{l}\text { Outcome } \\
\text { Measures }\end{array}$} & \multicolumn{5}{|c|}{ Emergence from PDoC $(n=23)$} & \multicolumn{5}{|c|}{ PDoC $(n=27)$} \\
\hline & \multirow[t]{2}{*}{ Event } & \multirow{2}{*}{$\begin{array}{l}\text { Median } \\
\text { achievement } \\
\text { time }(95 \% \mathrm{Cl} \text {, } \\
\text { days) }\end{array}$} & \multicolumn{3}{|c|}{ Cumulative probability } & \multirow[t]{2}{*}{ Event } & \multirow{2}{*}{$\begin{array}{l}\text { Median } \\
\text { achievement } \\
\text { time }(95 \% \mathrm{Cl} \text {, } \\
\text { days) }\end{array}$} & \multicolumn{3}{|c|}{ Cumulative probability } \\
\hline & & & 180 days & 365 days & 730 days & & & 180 days & 365 days & 730 days \\
\hline Auditory & 20 & $206(127,270)$ & 0.37 & 0.76 & 0.95 & 7 & NA $(534, N A)$ & 0.08 & 0.24 & 0.48 \\
\hline Visual & 16 & $269(127,400)$ & 0.42 & 0.61 & 0.92 & 10 & $614(252,614)$ & 0.15 & 0.39 & 0.51 \\
\hline Motor & 20 & $191(123,270)$ & 0.43 & 0.76 & 0.93 & 12 & $534(289,614)$ & 0.15 & 0.44 & 0.61 \\
\hline Oromotor & 17 & $284(154,593)$ & 0.36 & 0.61 & 0.76 & 11 & $614(274,1143)$ & 0.08 & 0.36 & 0.56 \\
\hline Communication & 14 & $239(117,406)$ & 0.37 & 0.68 & 0.92 & 4 & NA $(534, N A)$ & 0.04 & 0.14 & 0.26 \\
\hline Arousal & 18 & $231(127,437)$ & 0.38 & 0.65 & 0.90 & 5 & NA $(534, N A)$ & 0.04 & 0.19 & 0.30 \\
\hline
\end{tabular}

PDoC prolonged disorder of consciousness, NA not applicable

incorporating shorter lag time post-injury and the absence of intra-axial lesions best predicted the EDoC. The communication and auditory scores suggested a delayed but stronger correlation with EDoC compared with motor scores.

The strength of the study was that a wide range of clinical variables, including the whole subscales of CRS-R, were tracked longitudinally. In contrast to previous studies, we elucidated the course, predictive power, and effects of an extensive spectrum of neurobehavioral signs on the emergence from DOC, thus providing new insights into an optimal inpatient rehabilitation program that would best evaluate and maximize the potential for the recovery of consciousness. The merits of the methodology applied in our study were that the analysis of the full CRS-R performance profile, which includes all six subscale scores, enabled the accurate detection of conscious awareness [13]. Furthermore, our findings are supported by practice guidelines and updated recommendations for PDoC developed by the American Academy of Neurology, the American Congress of Rehabilitation Medicine, and the National Institute on Disability, Independent Living, and Rehabilitation Research. The findings suggest that clinicians should refer patients with $\mathrm{PDoC}$ to multidisciplinary rehabilitation teams with specialized training for optimal diagnostic and prognostic evaluations for further management, including effective medical monitoring and rehabilitative care. Prognostic counseling by clinicians should acknowledge that favorable outcomes and prognoses in patients with MCS diagnosed within 5 months of injury and traumatic etiology are variable [14].

Patients with non-traumatic injury exhibit a shorter window of recovery and greater disability than patients with TBI, and a majority of patients with traumatic injury regain consciousness within 12 months, and those with non-traumatic etiology by 3 months $[1,5,8]$. Nevertheless, our results showed that the recovery of patients with non-traumatic etiology may be prolonged. EDoC occurred in 217 (154, 345, range 64-1143) median days after non-traumatic injury and in 158 (124.25, 292.5, range 85-575) median days after TBI. These heterogeneous outcomes may be attributed to the Korean rehabilitation system, which allows intensive neurorehabilitation for both VS/UWS and MCS within 2 years of onset. Similar to previous studies, the prognosis was more favorable and heterogeneous for MCS than for VS/UWS and patients in MCS manifested EDoC in 209 (131.5, 346, range 64-1143) median days compared with $164(124,236.25$, range 112-345) median days in patients with VS/UWS $[11,12]$. Overall, the complexity of recovery outcomes in our study was consistent with recent findings reported in longitudinal studies of PDoC [4, 6-9, 15-19].

From a neurobehavioral perspective, our findings demonstrated that arousal and auditory functions were the most prognostic markers of emergence from PDoC. These findings were supported by higher levels of activation in the auditory association cortex using BOLD functional magnetic resonance imaging (fMRI) in response to a familiar voice speaking the patient's name, indicating factors associated with better prognosis [14, 20]. Furthermore, this study clinically supports previous reports suggesting that the level of auditory processing revealed by fMRI was strongly correlated with the 6-month outcome in each patient [21]. Di et al. reported earlier that the cerebral response to the patient's own name uttered by a familiar voice, which was measured with fMRI, might be a useful tool to preclinically distinguish minimally conscious states in a few patients behaviorally classified as vegetative [22].

At the level of functional connectivity, the auditory network is considered the most significant brain parameter distinguishing MCS from VS/UWS [23]. The regions of the auditory network comprising bilateral auditory and visual cortices are functionally connected in MCS more than in VS/UWS. The auditory-visual functional connectivity, also referred to as cross-modal interaction, is related to multisensory integration [24]. Multisensory integration has been suggested as a 


\section{a. Patients who emerged from PDoC}
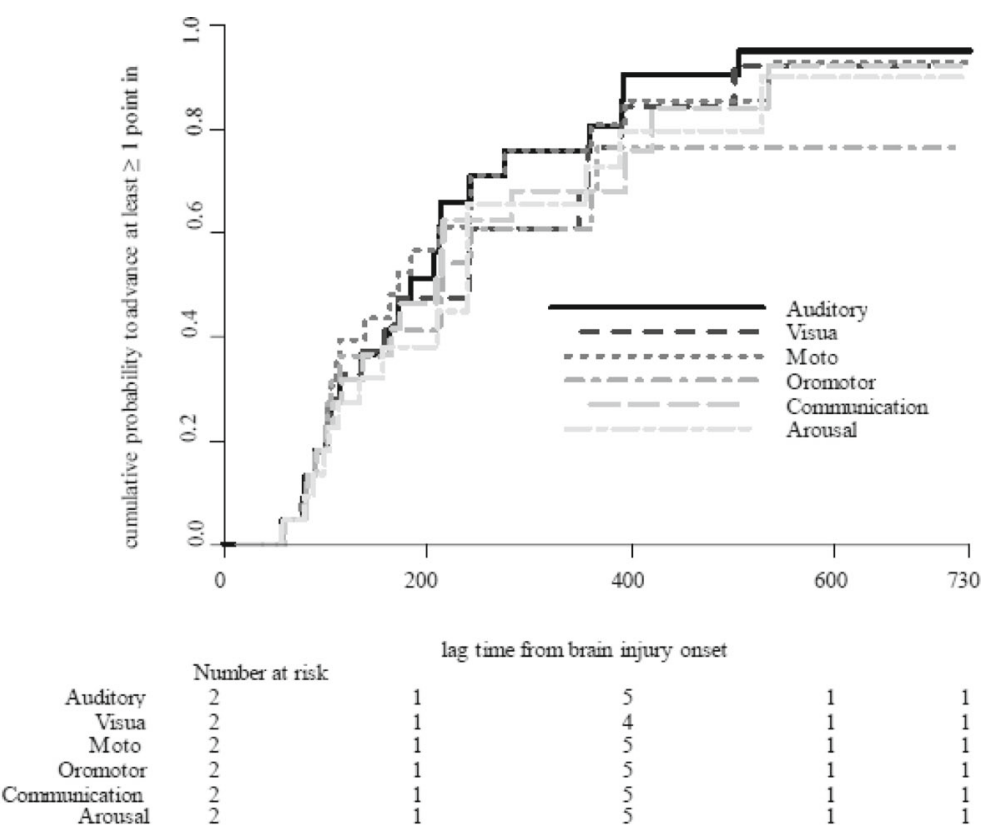

lag time from brain injury onset

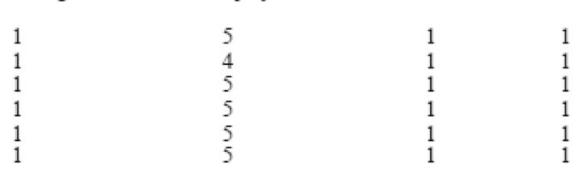

\section{b. Patients who persisted in PDoC}
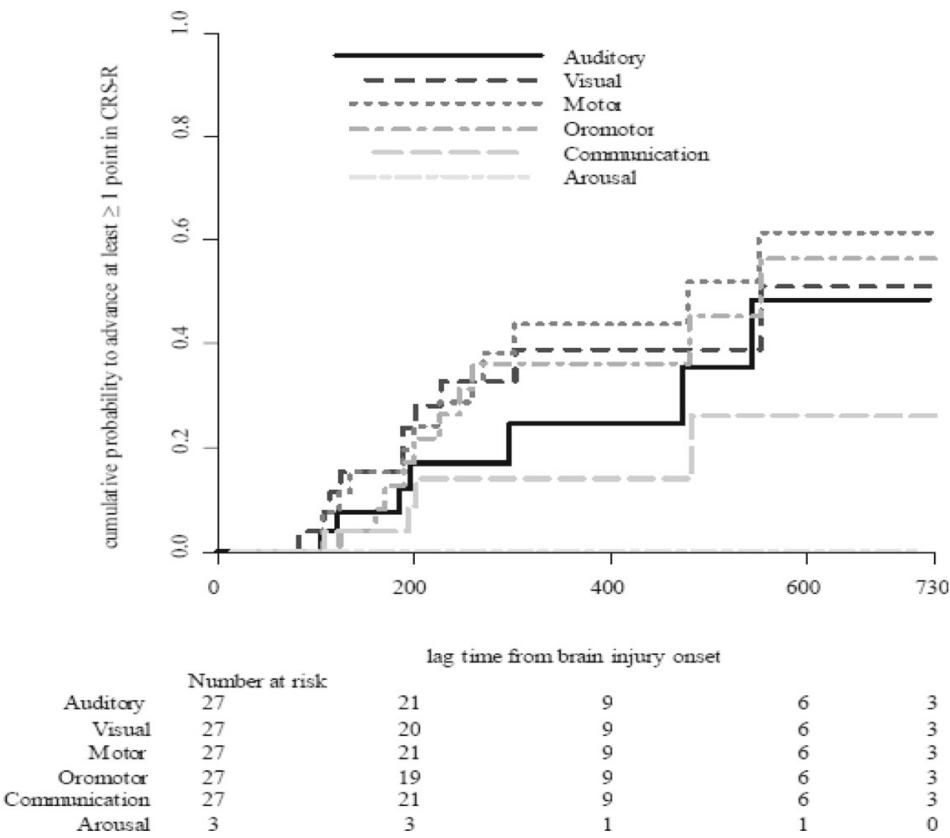

Fig. 2 Cumulative probability to reach at least 1-point advancement in the CRS-R. a Patients who emerged from PDoC. b Patients who persisted in $\mathrm{PDoC}$

facilitator in the top-down effects of higher-order regions, which may be necessary for conscious perception [25, 26]. Meanwhile, the cross-modal auditory-visual functional connectivity pairs are preserved in thalamocortical connectivity
[27]. Resumption of the functional relationship between thalami and associative cortices, such as prefrontal and anterior cingulate cortices, may lead to the restoration of consciousness, consistent with the behavioral expression 


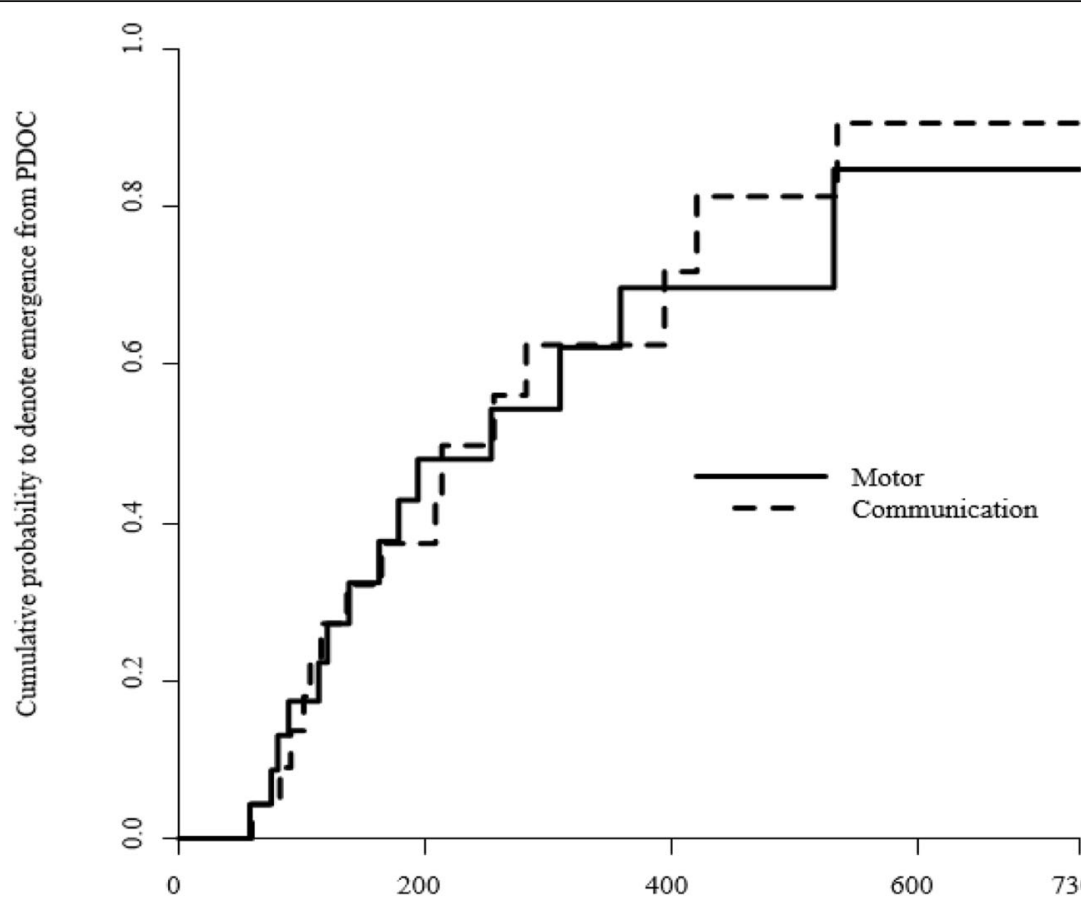

\begin{tabular}{cccccc} 
& \multicolumn{5}{c}{ lag time from brain injury onset } \\
Motor & Number at risk & 23 & 5 & 1 & 1 \\
Communication & 23 & 12 & 5 & 1 & 1
\end{tabular}

Fig. 3 Cumulative probability of EDoC in the CRS-R subscales. During the 180-day post-injury period, 39 and $32 \%$ of the patients in the recovery group manifested EDoC in motor and communication skills, respectively. Over time, the cumulative EDoC in the communication subscale increased and exceeded the motor subscale at 284 days post-injury. At 2 years post-injury, 88 and $93 \%$ of patients in the recovery group manifested EDoC in motor and communication skills, respectively. CRS-R, JFK Coma Recovery Scale-Revised; EDoC, emergence from disorder of consciousness; PDoC, prolonged disorder of consciousness

indicated by auditory or communication subscales in the CRS-R [28].

In a recently published cross-sectional multimodal imaging study analyzing the neural correlates in patients who emerged from MCS, the patients who emerged from MCS were characterized by a correlation between the networks and increased brain metabolism [29]. Further, novel behavioral correlates of auditory mismatch negativity event-related potentials (ERP) were detected in the auditory cortices [30].

It is worth mentioning that Giacino et al. tracked the recovery of six behavioral benchmarks derived from the CRS-R over a 6-week period during inpatient rehabilitation in patients with traumatic PDoC that extended four to 16 weeks post-injury [31]. The study revealed that patients in MCS with preserved language function were most likely to recover other high-level behaviors associated with functional recovery, analogous to the results in the present study. Moreover, members of the Traumatic Brain Injury Model Systems reported that a substantial number of patients with PDoC admitted to acute inpatient rehabilitation recovered independent functioning over as long as 5 years, especially if they followed commands before hospital discharge [6].

With regard to the temporal dynamics and cumulative recovery outcomes of the neurobehavioral profiles, our study revealed the highest probability of advanced motor function in the first 6 months, similar to early motor recovery in stroke patients, which primarily occurs within the first few months [32]. However, after 1 year, the auditory and communication functions also improved and showed the greatest cumulative probability of improvement in the 2 years post-injury. In this context, when the patient fails to show cortically driven behaviors, such as communication, during the first year after the brain injury, it is important to adopt further powerful approaches to identify cortical activity or 'volition without action' based on fMRI, as well as electroencephalography and ERP [33, 34].

Our results should be interpreted cautiously because of the small sample size and the limited number of patients investigated. Further, similar to all retrospective analyses, we could not control the assessment intervals of CRS-R that may have influenced the results. The CRS-R evaluation period varied from daily to every 6 weeks, with an average of 
monthly assessments. Even though the CRS-R has served as a useful tool for the differentiation between MCS and VS/ UWS with high reliability, validity, and sensitivity, spontaneous variability of the relevant neuronal or non-neuronal parameters over time in patients with severe disorder of consciousness may lead to spontaneous fluctuations [35, 36]. Hence, individual variability on the CRS-R may suggest limited diagnostic accuracy. Previous studies have reported high rates of misdiagnosis of $\mathrm{PDoC}$, reaching up to $40 \%[37,38]$.

Several studies have reported the beneficial effect of neuroimaging technologies, such as arterial spin labeling, magnetic resonance imaging, proton magnetic resonance spectroscopy, diffusion tensor imaging metrics, and voxel-based lesion-symptom mapping in the assessment of patients with severe brain injuries [39-43]. Future studies comprising more homogeneous and larger samples, with prospective and regular assessment of CRS-R, combined with neurotechnology-based assessments may corroborate our study findings.

Notwithstanding these limitations, our study facilitates clinician investigations of individuals with $\mathrm{PDoC}$ who can potentially benefit from inpatient rehabilitation and the establishment of optimal rehabilitation programs. Indeed, careful observation and evaluation of auditory perception and the facilitation of auditory responses may be important for successful outcomes.

\section{Conclusions}

Significant recovery of consciousness was observed in patients with PDoC during inpatient neurorehabilitation. The course and prediction of the recovery and the effects of neurobehavioral signs on the emergence from PDoC were elucidated in this study. In particular, careful evaluation of auditory perception and facilitation of the auditory response may be clinically important for the successful outcomes of neurorehabilitation in patients with PDoC.

\section{Supplementary information}

Supplementary information accompanies this paper at https://doi.org/10. 1186/s12883-020-01758-5.

\footnotetext{
Additional file 1. Demographic data. Demographic data including age ranges, sex, level of consciousness at admission, etiology, injury type, craniectomy, cranioplasty, CRS-R score at admission, hydrocephalus, presence of VP shunt, anti-epileptic drug, seizure, education, duration of disorder of consciousness, and emergence from DOC are described.

Additional file 2. CRS-R scores data. Total score and six neurobehavior domains based on the JFK Coma Recovery Scale-Revised (CRS-R) at admission and at discharge of enrolled patients are demonstrated.
}

\section{Abbreviations}

PDoC: Prolonged disorders of consciousness; EDoC: Emergence from prolonged disorders of consciousness; CRS-R: JFK Coma Recovery ScaleRevised; MCS: Minimally conscious state; DoC: Disorders of consciousness; VS/UWS: Vegetative state/unresponsive wakefulness syndrome; ABI: Acquired brain injury; CDW: Clinical Data Warehouse; TBI: Traumatic brain injury;
IQR: Interquartile range; HR: Hazard ratio; NA: Not applicable; fMRI: Functional magnetic resonance imaging; ERP: Event-related potentials

\section{Acknowledgments}

The Department of Biostatistics of the Catholic Research Coordinating Center, Catholic Medical Center, South Korea provided statistical assistance.

\section{Authors' contributions}

HYL developed the study concept, analyzed data, contributed to study design, and wrote the manuscript. TWK contributed to the study concept, data analysis, drafting of the manuscript, and project supervision. JHP analyzed the data and wrote the manuscript. ARK analyzed the data. MP conducted statistical analyses. All authors read and approved the manuscript. Permission was received from all authors of any "personal communications" cited in the article and all authors have agreed to conditions noted on the Authorship Agreement Form.

\section{Funding}

No funding was obtained for the preparation of this retrospective study.

\section{Availability of data and materials}

The datasets used and/or analyzed during the current study are available and deposited.

\section{Ethics approval and consent to participate}

The Methods section in the manuscript includes a statement that the Institutional Review Boards of the National Traffic Injury Rehabilitation Hospital approved our study. This study was a retrospective analysis and received approval from an ethical standards committee to conduct this study. Patient permission was not needed as the data used in this study were de-identified and anonymised upon collection and transferred to the research team.

\section{Consent for publication}

Patient's permission was not required as the data were de-identified using existing records. We omitted any identifying details regarding the patients from the manuscript. The article does not include any figure or video of a recognizable patient.

\section{Competing interests}

HYL, JHP, ARK, MP, and TWK declare that the research was conducted in the absence of any commercial or financial relationships that could be construed as a potential conflict of interest.

\section{Author details}

${ }^{1}$ TBI rehabilitation center, National Traffic Injury Rehabilitation Hospital, 260, Jungang-ro, Dogok-ri, Yangpyeong-eup, Yangpyeong-gun, Gyeonggi-do 12564, South Korea. ${ }^{2}$ Department of Rehabilitation Medicine, Seoul National University Hospital, Seoul University College of Medicine, Seoul, South Korea. ${ }^{3}$ Department of Medicine, the Graduate School of Yonsei University, Seoul, South Korea. ${ }^{4}$ Department of Rehabilitation Medicine, Gangnam Severance Hospital, Rehabilitation Institute of Neuromuscular Disease, Yonsei University College of Medicine, Seoul, South Korea. ${ }^{5}$ Department of Rehabilitation Medicine, School of Medicine, Kyungpook National University, Kyungpook National University Hospital, Daegu, South Korea. ${ }^{6}$ Department of Biostatistics, Clinical Research Coordinating Center, Catholic Medical Center The Catholic University of Korea, Seoul, Republic of Korea.

Received: 17 November 2019 Accepted: 3 May 2020

Published online: 20 May 2020

\section{References}

1. Ashwal S, Cranford R. Medical aspects of the persistent vegetative state--a correction. N Engl J Med. 1995;333(2):130.

2. Giacino JT, Ashwal S, Childs N, Cranford R, Jennett B, Katz DI, et al. The minimally conscious state: definition and diagnostic criteria. Neurology. 2002;58(3):349-53.

3. Laureys S, Celesia GG, Cohadon F, Lavrijsen J, León-Carrión J, Sannita WG, et al. Unresponsive wakefulness syndrome: a new name for the vegetative state or apallic syndrome. BMC Med. 2010;8(1):68. 
4. Katz DI, Polyak M, Coughlan D, Nichols M, Roche A. Natural history of recovery from brain injury after prolonged disorders of consciousness: outcome of patients admitted to inpatient rehabilitation with 1-4 year follow-up. Prog Brain Res. 2009;177:73-88.

5. Turner-Stokes L. Prolonged disorders of consciousness: new national clinical guidelines from the Royal College of Physicians, London. Clin Med. 2014; 14(1):4-5.

6. Whyte J, Nakase-Richardson R, Hammond FM, McNamee S, Giacino JT, Kalmar K, et al. Functional outcomes in traumatic disorders of consciousness: 5-year outcomes from the National Institute on Disability and Rehabilitation Research traumatic brain injury model systems. Arch Phys Med Rehabil. 2013;94(10):1855-60.

7. Nakase-Richardson R, Whyte J, Giacino JT, Pavawalla S, Barnett SD, Yablon SA, et al. Longitudinal outcome of patients with disordered consciousness in the NIDRR TBI model systems programs. J Neurotrauma. 2012;29(1):59-65.

8. Luaute J, Maucort-Boulch D, Tell L, Quelard F, Sarraf T, Iwaz J, et al. Longterm outcomes of chronic minimally conscious and vegetative states. Neurology. 2010;75(3):246-52.

9. Lammi MH, Smith VH, Tate RL, Taylor CM. The minimally conscious state and recovery potential: a follow-up study 2 to 5 years after traumatic brain injury. Arch Phys Med Rehabil. 2005;86(4):746-54

10. Shimamura N, Munakata A, Naraoka M, Ogasawara Y, Oyama K, Ohkuma H. Epidemiological investigation of patients in persistent vegetative states in Aomori, Japan. No Shinkei Geka. 2015;43(8):705-8.

11. Laureys S, Boly M. The changing spectrum of coma. Nat Rev Neurol. 2008; 4(10):544.

12. Giacino JT, Kalmar K, Whyte J. The JFK coma recovery scale-revised: measurement characteristics and diagnostic utility. Arch Phys Med Rehabil. 2004;85(12):2020-9.

13. Bodien YG, Carlowicz CA, Chatelle C, Giacino JT. Sensitivity and specificity of the coma recovery scale-revised total score in detection of conscious awareness. Arch Phys Med Rehabil. 2016;97(3):490-2.

14. Giacino JT, Katz DI, Schiff ND, Whyte J, Ashman EJ, Ashwal S, et al. Practice guideline update recommendations summary: disorders of consciousness: report of the guideline development, dissemination, and implementation Subcommittee of the American Academy of neurology; the American congress of rehabilitation medicine; and the National Institute on Disability, Independent Living, and Rehabilitation Research. Arch Phys Med Rehabil. 2018;99(9):1699-709.

15. Estraneo A, Moretta P, Loreto V, Lanzillo B, Santoro L, Trojano L. Late recovery after traumatic, anoxic, or hemorrhagic long-lasting vegetative state. Neurology. 2010;75(3):239-45.

16. Giacino J, Whyte J. The vegetative and minimally conscious states: current knowledge and remaining questions. J Head Trauma Rehabil. 2005;20(1):3050.

17. Klein A-M, Howell K, Vogler J, Grill E, Straube A, Bender A. Rehabilitation outcome of unconscious traumatic brain injury patients. J Neurotrauma. 2013;30(17):1476-83

18. Bernat JL. Chronic consciousness disorders. Annu Rev Med. 2009;60:381-92.

19. Whyte J, Katz D, Long D, DiPasquale MC, Polansky M, Kalmar K, et al. Predictors of outcome in prolonged posttraumatic disorders of consciousness and assessment of medication effects: a multicenter study. Arch Phys Med Rehabil. 2005;86(3):453-62.

20. Giacino JT, Katz DI, Schiff ND, Whyte J, Ashman EJ, Ashwal S, et al. Comprehensive systematic review update summary: disorders of consciousness: report of the guideline development, dissemination, and implementation Subcommittee of the American Academy of neurology; the American congress of rehabilitation medicine; and the National Institute on Disability, Independent Living, and Rehabilitation Research. Arch Phys Med Rehabil. 2018;99(9):1710-9.

21. Coleman MR, Davis MH, Rodd JM, Robson T, Ali A, Owen AM, et al. Towards the routine use of brain imaging to aid the clinical diagnosis of disorders of consciousness. Brain. 2009;132(9):2541-52.

22. Di H, Yu S, Weng X, Laureys S, Yu D, Li J, et al. Cerebral response to patient's own name in the vegetative and minimally conscious states. Neurology. 2007;68(12):895-9.

23. Demertzi A, Antonopoulos G, Heine L, Voss HU, Crone JS, de Los AC, et al. Intrinsic functional connectivity differentiates minimally conscious from unresponsive patients. Brain. 2015;138(9):2619-31.
24. Clavagnier S, Falchier A, Kennedy H. Long-distance feedback projections to area V1: implications for multisensory integration, spatial awareness, and visual consciousness. Cogn Affect Behav Neurosci. 2004;4(2):117-26.

25. Engel AK, Fries $P$, Singer W. Dynamic predictions: oscillations and synchrony in top-down processing. Nat Rev Neurosci. 2001;2(10):704.

26. Boly M, Garrido MI, Gosseries O, Bruno M-A, Boveroux P, Schnakers C, et al. Preserved feedforward but impaired top-down processes in the vegetative state. Science. 2011;332(6031):858-62.

27. Boveroux P, Vanhaudenhuyse A, Bruno M-A, Noirhomme Q, Lauwick S, Luxen $A$, et al. Breakdown of within-and between-network resting state functional magnetic resonance imaging connectivity during propofolinduced loss of consciousness. Anesthesiology. 2010;113(5):1038-53.

28. Laureys S, Faymonville M-E, Luxen A, Lamy M, Franck G, Maquet P. Restoration of thalamocortical connectivity after recovery from persistent vegetative state. Lancet. 2000;355(9217):1790-1.

29. Di Perri C, Bahri MA, Amico E, Thibaut A, Heine L, Antonopoulos G, et al. Neural correlates of consciousness in patients who have emerged from a minimally conscious state: a cross-sectional multimodal imaging study. Lancet Neurol. 2016;15(8):830-42.

30. El Karoui I, King J-R, Sitt J, Meyniel F, Van Gaal S, Hasboun D, et al. Eventrelated potential, time-frequency, and functional connectivity facets of local and global auditory novelty processing: an intracranial study in humans. Cereb Cortex. 2014;25(11):4203-12.

31. Giacino J, Sherer M, Bagiella E, Hammond F, Maurer-Karattup P. Behavioral and functional recovery in patients with prolonged traumatic disorders of consciousness. Arch Phys Med Rehabil. 2016;97(10):e3-4.

32. Hendricks HT, Van Limbeek J, Geurts AC, Zwarts MJ. Motor recovery after stroke: a systematic review of the literature. Arch Phys Med Rehabil. 2002; 83(11):1629-37.

33. Bruno M-A, Gosseries O, Ledoux D, Hustinx R, Laureys S. Assessment of consciousness with electrophysiological and neurological imaging techniques. Curr Opin Crit Care. 2011;17(2):146-51.

34. Laureys S, Schiff ND. Coma and consciousness: paradigms (re) framed by neuroimaging. Neuroimage. 2012;61(2):478-91.

35. Cortese MD, Riganello F, Arcuri F, Pugliese ME, Lucca LF, Dolce G, Sannita WG. Coma recovery scale-r: variability in the disorder of consciousness. BMC Neurol. 2015;15(1):186.

36. Han HJ, Kim EJ, Lee HJ, Pyun SB, Joa KL, Jung HY. Validation of Korean version of coma recovery scale-revised (K-CRSR). Ann Rehabil Med. 2018; 42(4):536.

37. Schnakers C, Vanhaudenhuyse A, Giacino J, Ventura M, Boly M, Majerus S, et al. Diagnostic accuracy of the vegetative and minimally conscious state: clinical consensus versus standardized neurobehavioral assessment. BMC Neurol. 2009;9(1):35

38. Majerus S, Gill-Thwaites H, Andrews K, Laureys S. Behavioral evaluation of consciousness in severe brain damage. Prog Brain Res. 2005;150:397-413.

39. Razek AAKA, Talaat M, El-Serougy L, Gaballa G, Abdelsalam M. Clinical applications of arterial spin labeling in brain tumors. J Comput Assist Tomogr. 2019;43(4):525-32.

40. Razek AAKA, Abdalla A, Ezzat A, Megahed A, Barakat T. Minimal hepatic encephalopathy in children with liver cirrhosis: diffusion-weighted MR imaging and proton MR spectroscopy of the brain. Neuroradiology. 2014; 56(10):885-91.

41. El-Serougy L, Abdel Razek AAK, Ezzat A, Eldawoody H, El-Morsy A. Assessment of diffusion tensor imaging metrics in differentiating low-grade from high-grade gliomas. Neuroradiol J. 2016;29(5):400-7.

42. Abdelrasoul AA, Elsebaie NA, Gamaleldin OA, Khalifa MH, Razek AAKA. Imaging of brain infarctions: beyond the usual territories. J Comput Assist Tomogr. 2019;43(3):443-51.

43. Lee KB, Lim SH. Prognosis and recovery of motor function with lesionsymptom mapping in patients with stroke. Brain Neurorehabil. 2016;10(1):e5.

\section{Publisher's Note}

Springer Nature remains neutral with regard to jurisdictional claims in published maps and institutional affiliations. 